\title{
Proposal and Evaluation of the Active Course Classification Support System with Exploitation-oriented Learning
}

\author{
Kazuteru Miyazaki and Masaaki Ida \\ National Institution for Academic Degrees and University Evaluation, \\ 1-29-1 Gakuennishimachi, Kodaira, Tokyo 187-8587, Japan, teru@niad.ac.jp \\ http://svrrd2.niad.ac.jp/faculty/teru/index.html
}

\begin{abstract}
The National Institution for Academic Degrees and University Evaluation (NIAD-UE) in Japan awards academic degrees based on the accumulation of credits. An applicant who wishes to be awarded a degree from NIAD-UE must classify his credits according to pre-determined criteria for each disciplinary field. The criteria are constructed by several items. A sub-committee of experts in the relevant field judges whether the course credit classification by the applicant is appropriate or not paying attention on the first item in the criteria. Recently, the Active Course Classification Support (ACCS) system has been proposed to support the course classification by the sub-committee. By being entered the classification item number into ACCS, ACCS indicates the course that belongs to the set of classification item number. However, the problem of deciding appropriate item numbers remains. This study aims to improve the method of determining item numbers, which should be judged by the sub-committee, by using machine learning. We use Exploitation-oriented Learning as the learning method for improving ACCS, and present a numerical example to show the effectiveness of our proposed method.
\end{abstract}

Keywords: reinforcement Learning, exploitation-oriented learning, course classification, recommender system

\section{Introduction}

Recently, there has been a wealth of impressive empirical results in Reinforcement Learning (RL)[13], as well as significant theoretical advances. Both types of advances in RL will lead to fruitful progress in real-world applications, such as decision making in social systems.

As globalization progresses, quality assurance for higher education is becoming an increasingly important issue in recent reforms of higher education worldwide. There is increasing demand for measuring the outcomes of student learning to improve the quality of higher education.

NIAD-UE(http://www.niad.ac.jp/) in Japan is an incorporated administrative agency of the Ministry of Education, Culture, Sports, Science and Technology (MEXT). The institution's mission is to contribute to the development 


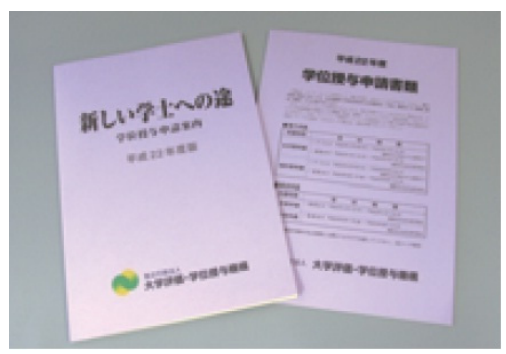

Fig. 1. The application guidebook "Alter- Fig. 2. The Committee for Validation and native Routes to a Bachelors Degree"

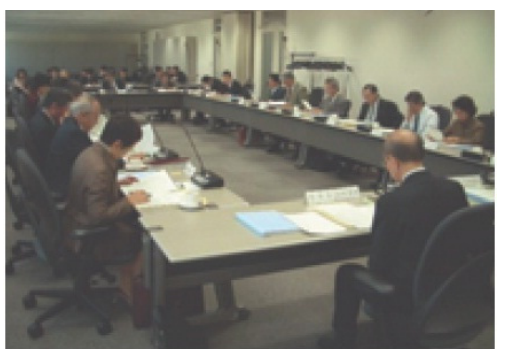

Examination of Degrees

Table 1. Criteria for the information engineering sub-field.

\begin{tabular}{|l|l|}
\hline Item 1 & Information Engineering Basic Theory (4+ credits) \\
Item 2 & Computer System (4+ credits) \\
Item 3 & Information Processing (4+ credits) \\
Item 4 & Electrical and Electronics, Communications, or Systems \\
\hline Item 5 & Exercises and Experiments in Information Engineering (6+ credits) \\
\hline Item 6 & Related Courses (4+ credits) \\
\hline Item 7 & Others \\
\hline
\end{tabular}

of higher education, in order to create a society in which the achievements of students at various higher education institutions, including non-university institutions, are duly appreciated. Assessing the results of various learning provided at the higher education level, NIAD-UE awards Bachelor's degrees to learners who have acquired sufficient academic credits and have made sufficient academic achievements in the particular disciplinary field.

In this degree-awarding process, an applicant who wishes to be awarded a degree from NIAD-UE must classify his earned credits according to pre-determined criteria for various disciplinary fields (Fig. 1). Table 1 is an example of the criteria. The validity of the applicant's course classification is judged by the subcommittee of the Committee for Validation and Examination of Degrees (Fig. 2) whose members are experts in the field. The members of the sub-committee take much time to read the syllabus of each course to judge whether the classification of the applicant is appropriate or not. NIAD-UE awards degrees to about 2,500 applicants each year, and to save labor, there is a need for using information technology to assist the course classification work. As a most promising method, application of RL in this classification process is strongly expected.

The Course Classification Support (CCS) system [5], which is a prototype recommender systems $[10,2]$, has been proposed to support the course classification. However, CCS has the drawback of excluding the occurrence frequency of technical terms, which plays a key role in the course classification. To overcome this problem, the Active Course Classification Support (ACCS) system has recently been proposed [6]. By using ACCS, we can control the number of 
candidates of courses that should be judged by the sub-committee, which would reduce the course classification workload for the sub-committee along the criteria for the chosen disciplinary field.

Each criterion for each disciplinary field is constructed by several items that characterize the field. In this paper, we discuss the criteria for the information engineering sub-field listed in Table 1. Items 1, 2, 3 and 4 are called specialized courses which are important for the information engineering sub-field, and are considered in this paper. Each item in specialized courses has several course examples to support the course classification, and usually has minimum number of credits that must be acquired by the applicant.

In the existing course classification process, the sub-committee usually starts judging the applicant's course classification based on the courses that are classified in the first item, for example, item 1 in Table 1. After checking the criteria of item 1, the sub-committee then checks the criteria of item 2 , and then those of item 3. Though the sub-committee has classified all specialized courses, we do not need to check the criteria of item 4 since it has no condition for the number of credits to be acquired by the applicant.

Generally, the difficulty of appropriate course classification depends on the disciplinary field. Therefore, in this paper, we aim to obtain the characteristics of course classification by machine learning, so that we can apply the results of the learning for the determining the item number and thus assist the judgments by the sub-committee. We use Exploitation-oriented Learning (XoL)[7], which is suitable in this situation as a machine learning method for ACCS, and present a numerical example to show the effectiveness of our method.

\section{Outline of the degree-awarding by NIAD-UE}

NIAD-UE's academic degree-awarding has two different schemes. One is based on a combination of the successful completion of a junior college program (or an equivalent level of education) and the accumulation of sufficient credits upon the completion of such a program or education level (Scheme I). The other scheme is based on the successful completion of an NIAD-UE-approved program provided by an educational institution that has been established legally, yet operates under the jurisdiction of ministries other than MEXT (Scheme II). A Bachelors degree can be obtained under either of these two schemes, whereas Masters and Doctoral degrees are awarded only under Scheme II. In this paper, we focus on Scheme I.

Although there exist similar systems as Scheme II in other countries, the Scheme I of NIAD-UE is a unique system in the world [8,9,11]. NIAD-UE annually publishes an application guidebook for Scheme I, Alternative Routes to a Bachelors Degree (ARBD) (Fig. 1). In this paper, we refer to the 2010 version which is available on NIAD-UE's Website at: http://www.niad.ac.jp/ICSFiles/afieldfile/2010/03/19/no7_5_gakushiH22.pdf

Under Scheme I, Bachelors degrees were awarded in 26 disciplinary fields in 2010, i.e. Art, Engineering, Literature, Nursing, Theology, and so on. Some dis- 
ciplinary fields are divided into sub-fields. For example, the engineering field has the following eight sub-fields: Mechanical Engineering, Electrical and Electronics Engineering, Information Engineering, Applied Chemistry, Biological Technology, Materials Engineering, Civil Engineering, and Architecture. In this paper, we focus on the information engineering sub-field in the engineering field.

An applicant has to certify that he has accumulated the appropriate number of credits according to the criteria pre-determined for each field. For example, the criteria for the information engineering sub-field are presented in Table 1. The course classification by an applicant is not always valid, and so the validity of course classification is judged by the sub-committee of the Committee for Validation and Examination of Degrees (Fig. 2), the members of which take much time to read the syllabus of each course. In recent years the number of applicants has been increasing, and so there is a need to assist the judging work of the sub-committee by using information technology. In the next section, we explain how CCS and ACCS are used to support course classification during degree-awarding by NIAD-UE.

\section{The Course Classification Support System}

\subsection{Construction of myDB}

The Course Classification Support (CCS) system receives syllabus data as an input and outputs a candidate course classification according to ARBD. It is important to construct a key database called the term-classification database to ensure an efficient course classification. In the following, we describe how to make myDB, which is one of the term-classification databases.

In 2002, the authors collected 962 syllabuses of information engineering courses offered by 13 universities in Japan [5]. They read these syllabuses and classified them into the items listed in Table 1. One syllabus may be classified into two or more of the items listed in the table.

Based on the classification, they constructed a set of special technical terms for each item, consisting of technical terms contained only in that item The technical terms are extracted by the method of reference [12]. This aims to find the characteristic terms that are contained only in each item. myDB is constructed by the set $\{\operatorname{myDB}(1), \ldots, \operatorname{myDB}(M)\}$ where $M$ is the number of items and $\operatorname{myDB}(i)(i=1,2, \ldots, M)$ is the set of special technical terms for item $i$.

\subsection{CCS and its Features}

CCS is a system that matches myDB and the syllabus data of each course. After matching between each term in the syllabus data and myDB, the matched terms and their corresponding item numbers are outputted.

The effectiveness of CCS in some examples has been confirmed [5]. However, it has the drawback of excluding the frequency of occurrence of technical terms, which plays a key role in the classification of courses. The Active Course Classification Support (ACCS) system [6] has been proposed to overcome this problem. 


\subsection{The Active Course Classification Support system}

myDB is a data set of terms by item that are present only in each item. However, the frequency of occurrence of technical terms is not considered. The specificity of a given technical term in a chosen item should change in a manner that is dependent on the frequency of the term's occurrence in syllabuses relevant to the item. Therefore, in reference [6], the authors reconfigured myDB by considering the frequency of occurrence of technical terms and incorporating this aspect into the CCS.

First, the authors extracted technical terms from all the syllabuses of the above-mentioned 13 universities by the method described in reference [12]. Subsequently, they carried out interval estimation of the probability that an extracted technical term is contained in item $i(i=1,2,3,4)$. In order to increase specificity, the upper and lower probability can be calculated as follows;

$$
\frac{\frac{x}{n}+\frac{Z_{\gamma}^{2}}{2 n} \pm \frac{Z_{\gamma}}{\sqrt{n}} \sqrt{\frac{x}{n}\left(1-\frac{x}{n}\right)+\frac{Z_{\gamma}^{2}}{4 n}}}{1+\frac{Z_{\gamma}^{2}}{n}}
$$

where $x$ is "the number of syllabuses that include the term and in which the term is contained in item $i$ ", $n$ is "the number of syllabuses that include the term", and $Z_{\gamma}$ is 1.96 and 2.58 at the confidence levels of $95 \%$ and $99 \%$, respectively. We set $Z_{\gamma}=1.96$ in Section 5 .

Taking this into account, they created a database that accumulates data sets of each technical term and the confidence interval estimated for the terms to be contained in item $i(i=1,2,3,4)$. Hereafter, this database is referred to as myDBc. myDBc is constructed by the set $\{m y D B c(1), \ldots, m y D B c(N)\}$ where $\operatorname{myDB} c(i)(i=1,2, \ldots, N)$ is a technical term and $N$ is the number of technical terms. Furthermore, we can get the lower and the upper limits of the confidence interval about the term $x$ on the item number $i(i=1,2, \ldots, M)$ by the functions $L L_{i}(m y D B c(x))$ and $U L_{i}(m y D B c(x))$, respectively.

In ACCS, myDB is replaced by myDBc. The paper [6] shows that we input syllabus data of the course submitted by the applicant into ACCS. ACCS matches terms ${ }^{1}$ between the syllabus data and myDBc. Then we can get "a matched technical term and the lower limit of the confidence interval of the probability that the term is contained in the item $i(i=1,2, \ldots, M)$." The subcommittee classifies the course in which the lower limit of the confidence interval about the item $i$ is greater than a thereshold value. If we gradually lower the threshold value from 1.0, we can identify those courses that are likely to be classified to the item number. We have called this method threshold learning.

\subsection{Features on ACCS}

The ACCS method can control the number of candidates of courses that should be judged by the sub-committee, thus reducing the workload for sub-committee members.

\footnotetext{
$\overline{{ }^{1} \text { In Section } 5}$, we attempt to input syllabus data directly into ACCS by scanning and OCR processing of the syllabuses.
} 
On the other hand, we do not understand the detail of how to decide the item number to be judged by the sub-committee. The sub-committee usually starts the judgment as to whether the course classification by an applicant is good based on the courses that are classified in the first item by the applicant. Ideally, it is desirable to learn the characteristics of the items that seem to be different for each field, and to determine the item number to be judged by the sub-committee. RL and XoL are machine learning methods that are suitable for such learning, so we examined combining them with ACCS.

\section{Proposal of ACCS with Exploitation-oriented Learning}

\subsection{Concept}

If the threshold learning about ACCS is perfect, that is, for example, the course that has the highest threshold value for an item number is actually classified to that item, then we can start from any item number. However, the threshold learning is not perfect, that is, the course that has the highest threshold value for an item number is not always classified to that item. For example, in the information engineering sub-field, though there are many courses belonging to Computer System (item 2), the number of courses belonging to Information Processing (item 3) tends to be smaller than item 2. Therefore, some courses that were regarded to be classified to item 3 by ACCS may be classified to item 2 by the sub-committee. This means that it is difficult to find courses belonging to Information Processing (item 3).

The incompleteness of threshold learning depends on the design of the criteria for each field. Therefore, in this paper, we aim to learn the characteristics of the criteria and to use it for our course classification. For example, in the information engineering sub-field, we aim to be able to find courses that belong to item 3 in preference to item 1 or 2 .

\subsection{Learning by Exploitation-oriented Learning}

We aim to learn the characteristics of the criteria for each field by RL or XoL, and to use them for determining the item number to be judged by the sub-committee. This means that we give ACCS a learning system that can determine the item number.

In XoL and RL, the learning agent senses a set of discrete attribute-value pairs and performs an action in some discrete varieties. The environment provides a reward signal to the agent as a result of some sequence of actions. A sensory input and an action constitute a pair that is termed a rule. The function that maps sensory inputs to actions is termed a policy. When there are $n$ sensory inputs and $m$ actions, we can make $n^{m}$ types of policies.

In many RL and some XoL methods, a scalar weight that indicates the importance of the rule is assigned to each rule. The learning progresses by updating the weight in the method. 


\section{Sensory Inputs and Actions}

We should change the item number to be judged depending on whether the requirements for each item are satisfied. We regard the satisfaction of requirements, that is, the number of credits required to satisfy the criteria, as sensory inputs. On the other hand, we regard the item number that we want to satisfy the criteria as an action output.

\section{Design of Rewards}

The design of a reward influences whether the learning is successful. The most basic reward design is to reward the learning agent when it has satisfied the criteria. However, if we use this design, we cannot expect to reduce the number of courses to be judged by the sub-committee, because the reward does not have information about the number of courses required to achieve the criteria. Therefore, in this paper, if the learning agent was able to achieve the criteria by the minimum number of courses, we will reward the learning agent.

\subsection{Overall Procedure of ACCS with XoL}

We show our proposal in the following and its framework in Fig. 3, which we call ACCS with XoL.

1. Fetch the required credit numbers for each item.

2. Determine the item number based on the policy that is learned by XoL or RL.

3. Submit the item number to ACCS as shown in the following.

(a) Matching with syllabus data of the course that is not classified by the sub-committee, determine a set $\mathrm{B}, B=\bigcup_{j=1}^{N_{t}}\left(m y D B c \cap S y l_{j}\right)$ where $S y l_{j}$ is the set of technical terms in the syllabus data of the course $j$ and $N_{t}$ is the number of courses that is not classified by the sub-committee.

(b) Select the term that has the highest $L L_{p}$ in the set $B$ where $p$ is the inputted item number $p$.

(c) Output the course that includes the term.

4. Obtain the course derived from ACCS.

5. Consult the sub-committee the classification of the course, and obtain the item number of the course from the sub-committee.

6. Recalculate the number of required credits for each item.

7. Go to step 1.

The learning agent corresponds to XoL or RL. It receives the number of required credits for each item as a sensory input, and selects and outputs an item number based on its policy as an action. ACCS receives the item number as an input, and outputs the course that has the highest value of the lower limit of the confidence interval for the item number to the sub-committee. The sub-committee classifies the course and outputs the item number of the course. We recalculate the number of required credits for each item. The learning agent receives a reward when the criteria have been achieved by the minimum number of courses. If the criteria for all items have been achieved, we can end the judgment of the applicant. 


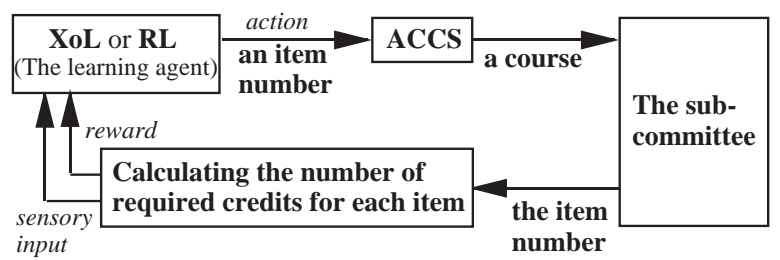

Fig. 3. ACCS with Exploitation-oriented Learning.

The learning agent selects an action based on the same probability for every action in every sensory input. This is called random walk (RA) and is generally used in XoL and RL, in order to improve a policy. The policy is saved by each individual applicant. If multiple policies are obtained from several applicants, we aim to integrate them by normalizing and accumulating their policies for each applicant's sensory input. The integrated and normalized policy is used to support the course classification of an unknown applicant. On the other hand, known classification results are used as learning data,

The output of the learning agent in Fig. 3 depends on the performance of ACCS. In general, the output of ACCS depends on many factors, such as the threshold value and so on, that are not included in a sensory input. This means that our task has the perceptual aliasing problem [1]. Therefore, many RL methods based on Dynamic Programming (DP) are not suitable for our task because they are designed to resolve Markov Decision Processes (MDPs).

\section{Evaluation of ACCS with XoL}

We show the effectiveness of ACCS with XoL in the information engineering sub-field. It has two steps. First, we aim to learn a policy, that is suitable for the sub-field, by XoL or RL. Next, we evaluate the learned policy combined with ACCS, which is ACCS with XoL.

\subsection{Learning a Policy by XoL or RL}

We must obtain policies for each sub-field in order to use ACCS with XoL. The policy's input is the number of required credits for each item and its output is an item number, as described in Section 4. In this paper, we aim to make a policy that is suitable for the information engineering sub-field, by XoL or RL.

We need to prepare learning data for XoL or RL. We use the syllabuses of the courses that were actually submitted for the information engineering subfield in fiscal year 2003 and 2004 as learning data to make the policy. They were submitted to NIAD-UE by four applicants independently, and were 48 courses of the science and engineering departments of University M, 28 courses of the engineering department of University K, 56 courses of the engineering science 
department of University $\mathrm{O}$ and 30 courses of the engineering department of University $\mathrm{H}$.

We prepare three types of learning method, that is Profit Sharing (PS)[3], Rational Policy Making algorithm (RPM)[4] and Q-learning (QL)[14]. PS and RPM are XoL methods, and QL is the most representative RL method. PS reinforces the scalar weights on rule sequence at the same time when it receives a reward. The rationality of PS was analyzed in reference [3]. RPM can achieve the same rationality without any scalar weight.

In PS, the initial weight is 0.0 and the reward is 100.0. PS depends on the design of the reinforcement function [3] which decides how to distribute a reward. We use the geometric decreasing function whose common ratio is $\frac{1}{4}$ that satisfies the rationality theorem in reference [3]. In QL, the initial weight is 10.0, the reward is 100.0 , the learning ratio is 0.8 , and the discount ratio is 0.9 . Those are decided by preliminary experiments.

For each learning method, we continued the learning until the criteria of the information engineering sub-field were satisfied 500,000 times for each applicant, which is equivalent to receiving a reward 2 million times. We only save the optimum policies as a result of learning ${ }^{2}$. The numbers of steps to receive a reward in the optimum policies were $8,8,25$, and 13 for universities $\mathrm{M}, \mathrm{K}, \mathrm{O}$ and $\mathrm{H}$, respectively.

\subsection{Experimental Results}

We aim to combine the learned policy in Section 5.1 with ACCS, namely, ACCS with XoL, and evaluate its performance. We use the syllabuses of the courses that were actually submitted from 2005 to 2010 by seven applicants (A to G) to evaluate the policy. We aim to reduce the number of courses required to satisfy the criteria, which thus reduces the number of courses to be judged by the sub-committee.

We show the number of courses required to satisfy the criteria by the three learning methods in Table 2. We conducted 100 trials with a different random seed for each applicant (A to G). Hence, after universities M, K, O and $\mathrm{H}$ have been learned by PS, RPM or QL, the courses of the seven applicants (A to G) are evaluated 100 times independently by the policy that has been learned for each learning method. Table 2 shows the average and standard deviation in parentheses for the 100 trials with different random seeds.

In the current classification, the sub-committee usually starts the judgment as to whether the course classification of an applicant is good based on the courses that are classified to item 1 in Table 1 by the applicant. After satisfying the criteria of item 1 , the sub-committee selects item 2 , and then item 3 . Though our target is all specialized courses from item 1 to 4 , we do not need to check the criteria of item 4 since it has no condition on the number of credits to be acquired by the applicant. Therefore, the current classification can be restricted

\footnotetext{
${ }^{2}$ Note that, in general, there are several types of optimum policies.
} 
Table 2. Results of each method.

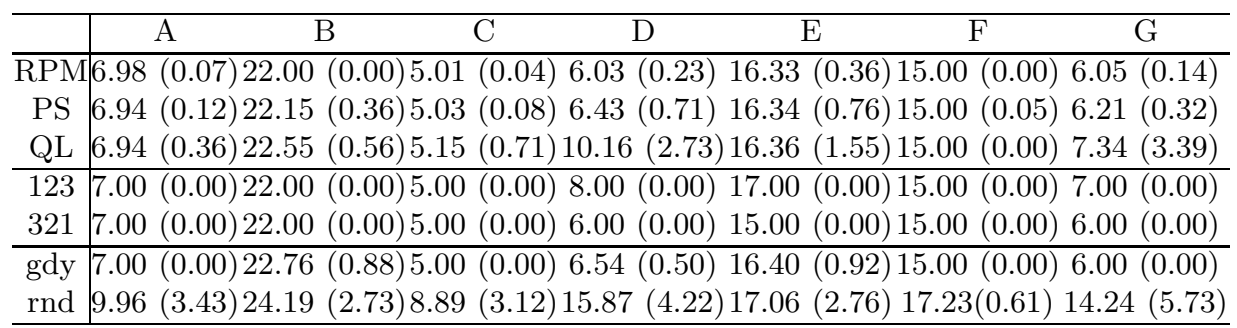

Table 3. Comparison with " 321 ".

\begin{tabular}{c|ccccccc}
\hline & $\mathrm{A}$ & $\mathrm{B}$ & $\mathrm{C}$ & $\mathrm{D}$ & $\mathrm{E}$ & $\mathrm{F}$ & $\mathrm{G}$ \\
\hline $\mathrm{RPM}$ & $(2.5,0.0)$ & $(0.0,0.0)$ & $(0.0,0.8)$ & $(0.0,0.7)$ & $(29.8,60.3)$ & $(0.0,0.0)$ & $(0.0,2.4)$ \\
PS & $(8.9,1.8)$ & $(0.0,13.7)$ & $(0.0,3.3)$ & $(0.0,27.6)$ & $(27.4,60.4)$ & $(0.0,0.5)$ & $(0.0,13.7)$ \\
QL & $(6.4,1.5)$ & $(0.0,34.5)$ & $(0.0,6.0)$ & $(0.0,82.0)$ & $(33.6,54.6)$ & $(0.0,0.0)$ & $(0.0,16.1)$ \\
\hline
\end{tabular}

to item 1 to 3 . We show the results of " 123 " in Table 2 where the sub-committee has judged courses in order of item number $1,2,3$.

Although we processed all combinations except "123", we only show the results for " 321 ", since it has the best performance among the six patterns "123","132"," 231 ","213"," 312 " and "321". In addition, "gdy", which means

Table 4. Summary of learning results. greedy, is the result when the sub-

\begin{tabular}{c|ccccccc}
\hline & $\mathrm{A}$ & $\mathrm{B}$ & $\mathrm{C}$ & $\mathrm{D}$ & $\mathrm{E}$ & $\mathrm{F}$ & $\mathrm{G}$ \\
\hline $\mathrm{RPM}$ & $>$ & $=+$ & $<$ & $=-$ & $=-$ & $=+$ & $=-$ \\
$\mathrm{PS}$ & $>$ & $=-$ & $<$ & $=-$ & $=-$ & $=+$ & $=-$ \\
$\mathrm{QL}$ & $>$ & $=-$ & $<$ & $<$ & $=-$ & $=+$ & $<$ \\
\hline
\end{tabular}
committee always checks the courses in the item which is furthest from the criteria. Also, "rnd" is the result when the sub-committee always checks the courses in the item decided on the same probability. Note that although " 321 " has the best performance, we cannot know the order in advance.

Table 3 compares the results with three learning methods and " 321 " in Table 2 . The values on the left of the parentheses in Table 3 are the percentages where the criteria can be achieved by the number of courses less than " 321 ". The values on the right of the parentheses are the percentages where it cannot improve on " 321 ".

The results of Table 2 are summarized in Table 4 where " $>$ " means that it can improve on " $321 "$, " $=+$ " means that it is equal to " $321 "$, "=-" means that it can improve on the worst case among the six patterns from " 123 " to " 312 ", and " $<$ " means that it cannot improve on the worst case. 


\subsection{Discussion}

Although the applicant $\mathrm{C}$ has " $<$ " for all methods in Table 4, the degree of deterioration is very small as shown in Table 3. Especially, in RPM, it is less than $1 \%$. Therefore, the result of applicant $\mathrm{C}$ is not considered to be a serious problem. In contrast, for applicant A, all methods have ">", which means that all methods have cases that can improve on the 7 courses that give the best performance in the current classification. The results are very significant since we can know better results than the known best. Although the same improvement is shown in applicant E, all methods have "=-" since it has a high percentage of deterioration.

QL is the worst among the three learning methods. Especially, applicant B and D show significant performance deterioration in Table 3. Though QL can guarantee the optimality in MDPs, it is not suitable for our task which goes beyond MDPs.

Furthermore, when several policies are combined, QL will receive another negative influence where it is possible to give the maximum weight for an item number that should not be learned. For example, in the sensory input where an insufficient number of courses are 4,4,3 for item 1,2,3, respectively, and the weights for each action 1,2,3 are " $0.5,0.45,0.05$ " for University $\mathrm{M}$ and " 0.2 , $0.3,0.35$ " for University $\mathrm{O}$, QL averages these weights to " $0.7,0.75,0.4$ ", that is " $0.38,0.40,0.22$ ". Therefore, it has a maximum weight for item 2 that should not be learned.

PS and RPM can guarantee rationality in some classes that go beyond MDPs ${ }^{3}$. Since our task belongs to the class, PS and RPM are more suitable for our task than QL. Like QL, PS can receive a negative influence when several policies are combined since it uses scalar weights for learning. However, the effect is less than that of QL, because PS is an XoL method, which strongly enhances the experience.

On the other hand, RPM does not use weights; it evaluates the rule by $\{0,1\}$ where "0" means bad and "1" means good. Therefore, unlike QL and PS, RPM is not negative influenced and can achieve the best performance.

\section{Conclusions}

The National Institution for Academic Degrees and University Evaluation in Japan awards awarding academic degrees for applicants based on the accumulation of course credits. An applicant's earned credits must be classified according to pre-determined criteria for his designated disciplinary field. Validation of his classification is carried out by a sub-committee of experts in the syllabus of each course. For assisting this course classification problem, we have already proposed the Active Course Classification Support (ACCS) system.

In this paper, we improved ACCS for course classification based on an Exploitation-oriented Learning mechanism. We presented numerical examples

\footnotetext{
${ }^{3}$ For details of the class refer to reference [4].
} 
for the specific disciplinary field, "Information Engineering" to show the effectiveness of our proposed method.

In the future, we will expand its applicability to various disciplinary fields

other than "information engineering", and will improve our system for practical use.

\section{References}

1. Chrisman, L.: Reinforcement learning with perceptual aliasing: The Perceptual Distinctions Approach. Proc. of the 10th National Conf. on Artificial Intelligence, 183-188 (1992)

2. Claypool, M., Gokhale, A., Miranda, T., Murnikov, P., Netes, D. and Sartin, M.: Combining content-based and collaborative filters in an online newspaper. Proc. of the ACM SIGIR '99 Workshop on Recommender Systems: Implementation and Evaluation, Berkeley, California, August (1999)

3. Miyazaki, K., Yamamura, M. and Kobayashi, S.: On the Rationality of Profit Sharing in Reinforcement Learning. Proc. of 3rd Int. Conf. on Fuzzy Logic, Neural Nets and Soft Computing, 285-288 (1994)

4. Miyazaki, K., and Kobayashi, S.: Learning Deterministic Policies in Partially Observable Markov Decision Processes. Proc. of 5th Int. Conf. on Intelligent Autonomous System, 250-257 (1998)

5. Miyazaki, K., Ida, M., Yoshikane, F., Nozawa, T. and Kita, H.: On development of a course classification support system using syllabus data. Computational Engineering I. 311-318 (2004)

6. Miyazaki, K., Ida, M., Yoshikane, F., Nozawa, T. and Kita, H.: Proposal of the Active Course Classification Support System to Support the Classification of Courses at the Degree-Awarding of NIAD-UE. Proc. of 6th Int. Symposium on Advanced Intelligent Systems, 685-690 (2005)

7. Miyazaki, K. and Kobayashi, S.: Exploitation-oriented Learning PS-r\#. J. of Advanced Computational Intelligence and Intelligent Informatics. 13(6), 624-630 (2009)

8. Mori, R.: The Credit Transfer System and the Validation Service at the Open University. Research in Academic Degrees. No.17, 183-198 (2003) (in Japanese)

9. Puirseil, S.: Quality Assurance in Irish Higher Education - The Higher Education and Training Awards Council. Research in Academic Degrees. 15, 124-140 (2001)

10. Resnick, P. and Varian, H.: Recommender Systems, introduction to special section of Communications of the ACM. March 1997, 40(3) (1997)

11. Tachi, T.: A Study on Thomas Edison State College, The External Degree College established by the State of New Jersey, Research in Academic Degrees. 10, 73-89 (1999) (in Japanese)

12. Yumoto, H., Mori, T. and Nakagawa, H.: Term Extraction Based on Occurrence and Concatenation Frequency. Natural Language Processing. 10(1), 27-45 (2003) (in Japanese)

13. Sutton, R. S. \& Barto, A. G.: Reinforcement Learning: An Introduction. A Bradford Book, MIT Press (1998)

14. Watkins, C. J. H. \& Dayan, P.: Technical note: Q-learning. Machine Learning. 8, 55-68 (1992) 